\title{
Research Article \\ Fixed Point Results for Generalized Contractive Multimaps in Metric Spaces
}

\author{
Abdul Latif ${ }^{1}$ and Afrah A. N. Abdou ${ }^{2}$ \\ ${ }^{1}$ Department of Mathematics, King Abdulaziz University, P.O. Box 80203, Jeddah 21589, Saudi Arabia \\ ${ }^{2}$ Girls College of Education, King Abdulaziz University, P.O. Box 14884, Jeddah 21434, Saudi Arabia
}

Correspondence should be addressed to Abdul Latif, latifmath@yahoo.com

Received 17 May 2009; Accepted 10 August 2009

Recommended by Mohamed A. Khamsi

The concept of generalized contractive multimaps in the setting of metric spaces is introduced, and the existence of fixed points for such maps is guaranteed under certain conditions. Consequently, our results either generalize or improve a number of fixed point results including the corresponding recent fixed point results of Ciric (2008), Latif-Albar (2008), Klim-Wardowski (2007), and Feng-Liu (2006). Examples are also given.

Copyright (C) 2009 A. Latif and A. A. N. Abdou. This is an open access article distributed under the Creative Commons Attribution License, which permits unrestricted use, distribution, and reproduction in any medium, provided the original work is properly cited.

\section{Introduction and Preliminaries}

Let $(X, d)$ be a metric space, $2^{X}$ a collection of nonempty subsets of $X, C B(X)$ a collection of nonempty closed bounded subsets of $X, C l(X)$ a collection of nonempty closed subsets of $X, K(X)$ a collection of nonempty compact subsets of $X$ and $H$ the Hausdorff metric induced by $d$. Then for any $A, B \in C B(X)$,

$$
H(A, B)=\max \left\{\sup _{x \in A} d(x, B), \sup _{y \in B} d(y, A)\right\},
$$

where $d(x, B)=\inf _{y \in B} d(x, y)$.

An element $x \in X$ is called a fixed point of a multivalued map $T: X \rightarrow 2^{X}$ if $x \in T(x)$. We denote $\operatorname{Fix}(T)=\{x \in X: x \in T(x)\}$. A sequence $\left\{x_{n}\right\}$ in $X$ is called an orbit of $T$ at $x_{0} \in X$ if $x_{n} \in T\left(x_{n-1}\right)$ for all $n \geq 1$.

A map $f: X \rightarrow \mathbb{R}$ is called lower semicontinuous if for any sequence $\left\{x_{n}\right\} \subset X$ with $x_{n} \rightarrow x \in X$ it implies that $f(x) \leq \liminf _{n \rightarrow \infty} f\left(x_{n}\right)$. 
Using the concept of Hausdorff metric, Nadler [1] established the following fixed point result for multivalued contraction maps, known as Nadler's contraction principle which in turn is a generalization of the well-known Banach contraction principle.

Theorem 1.1 (see [1]). Let $(X, d)$ be a complete metric space and let $T: X \rightarrow C B(X)$ be a contraction map. Then $\operatorname{Fix}(T) \neq \emptyset$.

Using the concept of the Hausdorff metric, many authors have generalized Nadler's contraction principle in many directions. But, in fact for most cases the existence part of the results can be proved without using the concept of Hausdorff metric. Recently, Feng and Liu [2] extended Nadler's fixed point theorem without using the concept of Hausdorff metric. They proved the following result.

Theorem 1.2. Let $(X, d)$ be a complete metric space and let $T: X \rightarrow C l(X)$ be a map such that for any fixed constants $h, b \in(0,1), h<b$, and for each $x \in X$ there is $y \in T(x)$ satisfying the following conditions:

$$
\begin{aligned}
& b d(x, y) \leq d(x, T(x)) \\
& d(y, T(y)) \leq h d(x, y) .
\end{aligned}
$$

Then $\operatorname{Fix}(T) \neq \emptyset$ provided a real-valued function $g$ on $X, g(x)=d(x, T(x))$ is lower semicontinuous. two results.

Recently, Klim and Wardowski [3] generalized Theorem 1.2 and proved the following

Theorem 1.3. Let $(X, d)$ be a complete metric space and let $T: X \rightarrow C l(X)$. Assume that the following conditions hold:

(i) there exist a number $b \in(0,1)$ and a function $k:[0, \infty) \rightarrow[0, b)$ such that for each $t \in[0, \infty)$,

$$
\limsup _{r \rightarrow t^{+}} k(r)<b
$$

(ii) for any $x \in X$ there is $y \in T(x)$ satisfying

$$
\begin{gathered}
b d(x, y) \leq d(x, T(x)), \\
d(y, T(y)) \leq k(d(x, y)) d(x, y) .
\end{gathered}
$$

Then $\operatorname{Fix}(T) \neq \emptyset$ provided a real-valued function $g$ on $X, g(x)=d(x, T(x))$ is lower semicontinuous.

Theorem 1.4. Let $(X, d)$ be a complete metric space and let $T: X \rightarrow K(X)$. Assume that the following conditions hold:

(i) there exists a function $k:[0, \infty) \rightarrow[0,1)$ such that for each $t \in[0, \infty)$,

$$
\limsup _{r \rightarrow t^{+}} k(r)<1,
$$


Fixed Point Theory and Applications

(ii) for any $x \in X$ there is $y \in T(x)$ satisfying

$$
\begin{gathered}
d(x, y)=d(x, T(x)), \\
d(y, T(y)) \leq k(d(x, y)) d(x, y) .
\end{gathered}
$$

Then $\operatorname{Fix}(T) \neq \emptyset$ provided a real-valued function $g$ on $X, g(x)=d(x, T(x))$ is lower semicontinuous.

Note that Theorem 1.3 generalizes Nadler's contraction principle and Theorem 1.2. Most recently, Ciric [4] obtained some interesting fixed point results which extend and generalize the cited results. Namely, [4, Theorem 5] generalizes [5, Theorem 5], [4, Theorem 6] generalizes [4, Theorems 1.2, 1.3], and [3, theorem 7] generalizes Theorem 1.4.

In [6], Kada et al. introduced the concept of $w$-distance on a metric space as follows:

A function $\omega: X \times X \rightarrow[0, \infty)$ is called $w$-distance on $X$ if it satisfies the following for each $x, y, z \in X$ :

$\left(w_{1}\right) \omega(x, z) \leq \omega(x, y)+\omega(y, z)$

$\left(w_{2}\right)$ a map $\omega(x, \cdot): X \rightarrow[0, \infty)$ is lower semicontinuous; that is, if a sequence $\left\{y_{n}\right\}$ in $X$ with $y_{n} \rightarrow y \in X$, then $\omega(x, y) \leq \liminf _{n \rightarrow \infty} \omega\left(x, y_{n}\right)$;

$\left(w_{3}\right)$ for any $\epsilon>0$, there exists $\delta>0$ such that $\omega(z, x) \leq \delta$ and $\omega(z, y) \leq \delta$ imply $d(x, y) \leq \epsilon$.

Note that, in general for $x, y \in X, \omega(x, y) \neq \omega(y, x)$ and not either of the implications $\omega(x, y)=0 \Leftrightarrow x=y$ necessarily hold. Clearly, the metric $d$ is a $w$-distance on $X$. Let $(Y,\|\cdot\|)$ be a normed space. Then the functions $\omega_{1}, \omega_{2}: Y \times Y \rightarrow[0, \infty)$ defined by $\omega_{1}(x, y)=\|y\|$ and $\omega_{2}(x, y)=\|x\|+\|y\|$ for all $x, y \in Y$ are $w$-distances [6]. Many other examples and properties of the $w$-distance can be found in [6,7].

The following lemma is crucial for the proofs of our results.

Lemma 1.5 (see [8]). Let $K$ be a closed subset of $X$ and $\omega$ be a w-distance on $X$. Suppose that there exists $u \in X$ such that $\omega(u, u)=0$. Then $\omega(u, K)=0 \Leftrightarrow u \in K$, where $\omega(u, K)=\inf _{y \in K} \omega(u, y)$. as follows.

Most recently, the authors of this paper generalized Latif and Albar [9, Theorem 1.3]

Theorem 1.6 (see $[10])$. Let $(X, d)$ be a complete metric space with a w-distance $\omega$. Let $T: X \rightarrow$ $\mathrm{Cl}(\mathrm{X})$ be a multivalued map satisfying that for any constant $b \in(0,1)$ and for each $x \in X$ there is $y \in J_{b}^{x}$ such that

$$
\omega(y, T(y)) \leq k(\omega(x, y)) \omega(x, y)
$$

where $J_{b}^{x}=\{y \in T(x): b \omega(x, y) \leq \omega(x, T(x))\}$ and $k$ is a function from $[0, \infty)$ to $[0, b)$ with $\lim \sup _{r \rightarrow t^{+}} k(r)<b$, for every $t \in[0, \infty)$. Suppose that a real-valued function $g$ on $X$ defined by $g(x)=\omega(x, T(x))$ is lower semicontinuous. Then there exists $v_{o} \in X$ such that $g\left(v_{o}\right)=0$. Further, if $\omega\left(v_{o}, v_{o}\right)=0$, then $v_{0} \in \operatorname{Fix}(T)$.

The aim of this paper is to present some more general results on the existence of fixed points for multivalued maps satisfying certain conditions. Our results unify and generalize 
the corresponding results of Mizoguchi and Takahashi [5], Klim and Wardowski [3], Latif and Abdou [10], Ciric [4], Feng and Liu [2], Latif and Albar [9] and several others.

\section{The Results}

First we prove a theorem which is a generalization of Ciric [4, Theorem 5] and due to Klim and Wardowski [3, Theorem 1.4].

Theorem 2.1. Let $(X, d)$ be a complete metric space with a w-distance $\omega$. Let $T: X \rightarrow C l(X)$ be a multivalued map. Assume that the following conditions hold:

(i) there exists a function $\varphi:[0, \infty) \rightarrow[0,1)$ such that for each $t \in[0, \infty)$

$$
\limsup _{r \rightarrow t^{+}} \varphi(r)<1
$$

(ii) for any $x \in X$, there exists $y \in T(x)$ satisfying

$$
\begin{gathered}
\omega(x, y) \leq(2-\varphi(\omega(x, y))) \omega(x, T(x)), \\
\omega(y, T(y)) \leq \varphi(\omega(x, y)) \omega(x, y)
\end{gathered}
$$

(iii) the map $f: X \rightarrow \mathbb{R}$, defined by $f(x)=\omega(x, T(x))$ is lower semicontinuous.

Then there exists $v_{0} \in X$ such that $f\left(v_{0}\right)=0$. Further if $\omega\left(v_{0}, v_{0}\right)=0$, then $v_{0} \in T\left(v_{0}\right)$.

Proof. let $x_{0} \in X$ be any initial point. Then there exists $x_{1} \in T\left(x_{0}\right)$ such that

$$
\begin{gathered}
\omega\left(x_{0}, x_{1}\right) \leq\left(2-\varphi\left(\omega\left(x_{0}, x_{1}\right)\right)\right) \omega\left(x_{0}, T\left(x_{0}\right)\right) \\
\omega\left(x_{1}, T\left(x_{1}\right)\right) \leq \varphi\left(\omega\left(x_{0}, x_{1}\right)\right) \omega\left(x_{0}, x_{1}\right) .
\end{gathered}
$$

From (2.3) we get

$$
\omega\left(x_{1}, T\left(x_{1}\right)\right) \leq \varphi\left(\omega\left(x_{0}, x_{1}\right)\right)\left(2-\varphi\left(\omega\left(x_{0}, x_{1}\right)\right)\right) \omega\left(x_{0}, T\left(x_{0}\right)\right) .
$$

Define a function $\psi:[0, \infty) \rightarrow[0, \infty)$ by

$$
\psi(t)=\varphi(t)(2-\varphi(t))=1-(1-\varphi(t))^{2} .
$$

Using the facts that for each $t \in[0, \infty), \varphi(t)<1$ and $\lim _{r \rightarrow t^{+}} \sup \varphi(r)<1$, we have

$$
\begin{gathered}
\psi(t)<1, \\
\limsup _{r \rightarrow t^{+}} \psi(r)<1 \quad \forall t \in[0, \infty)
\end{gathered}
$$


Fixed Point Theory and Applications

From (2.4) and (2.5), we have

$$
\omega\left(x_{1}, T\left(x_{1}\right)\right) \leq \psi\left(\omega\left(x_{0}, x_{1}\right)\right) \omega\left(x_{0}, T\left(x_{0}\right)\right) .
$$

Similarly, for $x_{1} \in X$, there exists $x_{2} \in T\left(x_{1}\right)$ such that

$$
\begin{gathered}
\omega\left(x_{1}, x_{2}\right) \leq\left(2-\varphi\left(\omega\left(x_{1}, x_{2}\right)\right)\right) \omega\left(x_{1}, T\left(x_{1}\right)\right) \\
\omega\left(x_{2}, T\left(x_{2}\right)\right) \leq \varphi\left(\omega\left(x_{1}, x_{2}\right)\right) \omega\left(x_{1}, x_{2}\right) .
\end{gathered}
$$

Thus

$$
\omega\left(x_{2}, T\left(x_{2}\right)\right) \leq \psi\left(\omega\left(x_{1}, x_{2}\right)\right) \omega\left(x_{1}, T\left(x_{1}\right)\right)
$$

Continuing this process we can get an orbit $\left\{x_{n}\right\}$ of $T$ in $X$ satisfying the following:

$$
\begin{aligned}
\omega\left(x_{n}, x_{n+1}\right) & \leq\left(2-\varphi\left(\omega\left(x_{n}, x_{n+1}\right)\right)\right) \omega\left(x_{n}, T\left(x_{n}\right)\right), \\
\omega\left(x_{n+1}, T\left(x_{n+1}\right)\right) & \leq \psi\left(\omega\left(x_{n}, x_{n+1}\right)\right) \omega\left(x_{n}, T\left(x_{n}\right)\right),
\end{aligned}
$$

for each integer $n \geq 0$. Since $\psi(t)<1$ for each $t \in[0, \infty)$ and from (2.12), we have for all $n \geq 0$

$$
\omega\left(x_{n+1}, T\left(x_{n+1}\right)\right)<\omega\left(x_{n}, T\left(x_{n}\right)\right) .
$$

Thus the sequence of nonnegative real numbers $\left\{\omega\left(x_{n}, T\left(x_{n}\right)\right)\right\}$ is decreasing and bounded below, thus convergent. Therefore, there is some $\delta \geq 0$ such that

$$
\lim _{n \rightarrow \infty} \omega\left(x_{n}, T\left(x_{n}\right)\right)=\delta
$$

From (2.11), as $\varphi(t)<1$ for all $t \geq 0$, we get

$$
\omega\left(x_{n}, T\left(x_{n}\right)\right) \leq \omega\left(x_{n}, x_{n+1}\right)<2 \omega\left(x_{n}, T\left(x_{n}\right)\right),
$$

Thus, we conclude that the sequence of nonnegative reals $\left\{\omega\left(x_{n}, x_{n+1}\right)\right\}$ is bounded. Therefore, there is some $\theta \geq 0$ such that

$$
\liminf _{n \rightarrow \infty} \omega\left(x_{n}, x_{n+1}\right)=\theta
$$

Note that $\omega\left(x_{n}, x_{n+1}\right) \geq \omega\left(x_{n}, T\left(x_{n}\right)\right)$ for each $n \geq 0$, so we have $\theta \geq \delta$. Now we will show that $\theta=\delta$. Suppose that $\delta=0$. Then we get

$$
\lim _{n \rightarrow \infty} \omega\left(x_{n}, x_{n+1}\right)=0
$$


Now consider $\delta>0$. Suppose to the contrary, that $\theta>\delta$. Then $\theta-\delta>0$ and so from (2.14) and (2.16) there is a positive integer $n_{0}$ such that

$$
\begin{gathered}
\omega\left(x_{n}, T\left(x_{n}\right)\right)<\delta+\frac{\theta-\delta}{4} \quad \forall n \geq n_{0}, \\
\theta-\frac{\theta-\delta}{4}<\omega\left(x_{n}, x_{n+1}\right) \quad \forall n \geq n_{0} .
\end{gathered}
$$

Then from (2.19), (2.11) and (2.18), we get

$$
\begin{aligned}
\theta-\frac{\theta-\delta}{4} & <\omega\left(x_{n}, x_{n+1}\right) \\
& \leq\left(2-\varphi\left(\omega\left(x_{n}, x_{n+1}\right)\right)\right) \omega\left(x_{n}, T\left(x_{n}\right)\right) \\
& <\left(2-\varphi\left(\omega\left(x_{n}, x_{n+1}\right)\right)\right)\left[\delta+\frac{\theta-\delta}{4}\right]
\end{aligned}
$$

Thus for all $n \geq n_{0}$,

$$
\left(2-\varphi\left(\omega\left(x_{n}, x_{n+1}\right)\right)\right)>\frac{3 \theta+\delta}{3 \delta+\theta}
$$

that is,

$$
1+\left(1-\varphi\left(\omega\left(x_{n}, x_{n+1}\right)\right)\right)>1+\frac{2(\theta-\delta)}{3 \delta+\theta}
$$

and we get

$$
-\left(1-\varphi\left(\omega\left(x_{n}, x_{n+1}\right)\right)\right)^{2}<-\left[\frac{2(\theta-\delta)}{3 \delta+\theta}\right]^{2}
$$

Thus for all $n \geq n_{0}$,

$$
\begin{aligned}
\psi\left(\omega\left(x_{n}, x_{n+1}\right)\right) & =1-\left(1-\varphi\left(\omega\left(x_{n}, x_{n+1}\right)\right)\right)^{2} \\
& <1-\left[\frac{2(\theta-\delta)}{3 \delta+\theta}\right]^{2}
\end{aligned}
$$

Thus, from (2.12) and (2.24), we get

$$
\omega\left(x_{n+1}, T\left(x_{n+1}\right)\right) \leq h \omega\left(x_{n}, T\left(x_{n}\right)\right) \quad \forall n \geq n_{0},
$$


where $h=1-[2(\theta-\delta) /(3 \delta+\theta)]^{2}$. Clearly $h<1$ as $\theta>\delta$. From (2.18) and (2.25), we have for any $k \geq 1$

$$
\omega\left(x_{n_{0}+k}, T\left(x_{n_{0}+k}\right)\right) \leq h^{k} \omega\left(x_{n_{0}}, T\left(x_{n_{0}}\right)\right) .
$$

Since $\delta>0$ and $h<1$, there is a positive integer $k_{0}$ such that $h^{k_{0}} \omega\left(x_{n_{0}}, T\left(x_{n_{0}}\right)\right)<\delta$. Now, since $\delta \leq \omega\left(x_{n}, T\left(x_{n}\right)\right)$ for each $n \geq 0$, by (2.26) we have

$$
\delta \leq \omega\left(x_{n_{0}+k_{0}}, T\left(x_{n_{0}+k_{0}}\right)\right) \leq h^{k_{0}} \omega\left(x_{n_{0}}, T\left(x_{n_{0}}\right)\right)<\delta .
$$

a contradiction. Hence, our assumption $\theta>\delta$ is wrong. Thus $\delta=\theta$. Now we will show that $\theta=0$. Since $\theta=\delta \leq \omega\left(x_{n}, T\left(x_{n}\right)\right) \leq \omega\left(x_{n}, x_{n+1}\right)$, then from (2.16) we can read as

$$
\liminf _{n \rightarrow \infty} \omega\left(x_{n}, x_{n+1}\right)=\theta+
$$

so, there exists a subsequence $\left\{\omega\left(x_{n_{k}}, x_{n_{k}+1}\right)\right\}$ of $\left\{\omega\left(x_{n}, x_{n+1}\right)\right\}$ such that

$$
\lim _{k \rightarrow \infty} \omega\left(x_{n_{k}}, x_{n_{k}+1}\right)=\theta+
$$

Now from (2.7) we have

$$
\limsup _{\omega\left(x_{n_{k}}, x_{n_{k}+1}\right) \rightarrow \theta+} \psi\left(\omega\left(x_{n_{k}}, x_{n_{k}+1}\right)\right)<1
$$

and from (2.12), we have

$$
\omega\left(x_{n_{k}}, T\left(x_{n_{k}+1}\right)\right) \leq \psi\left(\omega\left(x_{n_{k}}, x_{n_{k}+1}\right)\right) \omega\left(x_{n_{k}}, T\left(x_{n_{k}}\right)\right)
$$

Taking the limit as $k \rightarrow \infty$ and using (2.14), we get

$$
\begin{aligned}
\delta & =\limsup _{k \rightarrow \infty} \omega\left(x_{n_{k+1}}, T\left(x_{n_{k}+1}\right)\right) \\
& \leq\left(\limsup _{k \rightarrow \infty} \psi\left(\omega\left(x_{n_{k+1}}, x_{n_{k}+1}\right)\right)\right)\left(\limsup _{k \rightarrow \infty} \omega\left(x_{n_{k}}, T\left(x_{n_{k}}\right)\right)\right) \\
& =\left(\limsup _{\omega\left(x_{n_{k}}, x_{n_{k}+1}\right) \rightarrow \theta+} \psi\left(\omega\left(x_{n_{k}}, x_{n_{k}+1}\right)\right)\right) \delta .
\end{aligned}
$$

If we suppose that $\delta>0$, then from last inequality, we have

$$
\limsup _{\omega\left(x_{n_{k}}, x_{n_{k}+1}\right) \rightarrow \theta+} \psi\left(\omega\left(x_{n_{k}}, x_{n_{k}+1}\right)\right) \geq 1
$$


which contradicts with (2.30). Thus $\delta=0$. Then from (2.14) and (2.15), we have

$$
\lim _{n \rightarrow \infty} \omega\left(x_{n}, T\left(x_{n}\right)\right)=0+
$$

and thus

$$
\lim _{n \rightarrow \infty} \omega\left(x_{n}, x_{n+1}\right)=0+
$$

Now, let

$$
\alpha=\lim _{\omega\left(x_{n_{k}}, x_{n_{k}+1}\right) \rightarrow 0^{+}} \sup \psi\left(\omega\left(x_{n_{k}}, x_{n_{k}+1}\right)\right) .
$$

Then by (2.7), $\alpha<1$. Let $q$ be such that $\alpha<q<1$. Then there is some $n_{0} \in \mathbb{N}$ such that

$$
\psi\left(\omega\left(x_{n}, x_{n+1}\right)\right)<q \quad \forall n \geq n_{0} .
$$

Thus it follows from (2.12),

$$
\omega\left(x_{n+1}, T\left(x_{n+1}\right)\right) \leq q \omega\left(x_{n}, T\left(x_{n}\right)\right) \quad \forall n \geq n_{0} .
$$

By induction we get

$$
\omega\left(x_{n+1}, T\left(x_{n+1}\right)\right) \leq q^{n+1-n_{0}} \omega\left(x_{n_{0}}, T\left(x_{n_{0}}\right)\right) \quad \forall n \geq n_{0} .
$$

Now, using (2.15) and (2.39), we have

$$
\omega\left(x_{n}, x_{n+1}\right) \leq 2 q^{n-n_{0}} \omega\left(x_{n_{0}}, T\left(x_{n_{0}}\right)\right) \quad \forall n \geq n_{0} .
$$

Now, we show that $\left\{x_{n}\right\}$ is a Cauchy sequence, for all $m>n \geq n_{0}$, we get

$$
\begin{aligned}
\omega\left(x_{n}, x_{m}\right) & \leq \sum_{k=n}^{m-1} \omega\left(x_{k}, x_{k+1}\right) \\
& \leq 2 \sum_{k=n}^{m-1} q^{k-n_{0}} \omega\left(x_{n_{0}}, T\left(x_{n_{0}}\right)\right) \\
& \leq 2\left(\frac{q^{n-n_{0}}}{1-q}\right) \omega\left(x_{n_{0}}, T\left(x_{n_{0}}\right)\right) .
\end{aligned}
$$

Hence we conclude, as $q<1$, that $\left\{x_{n}\right\}$ is Cauchy sequence. Due to the completeness of $X$, there exists some $v_{0} \in X$ such that $\lim _{n \rightarrow \infty} x_{n}=v_{0}$. Since $f$ is lower semicontinuous and from (2.34), we have

$$
0 \leq f\left(v_{0}\right) \leq \liminf _{n \rightarrow \infty} f\left(x_{n}\right)=\omega\left(x_{n}, T\left(x_{n}\right)\right)=0,
$$


and thus, $f\left(v_{0}\right)=\omega\left(v_{0}, T\left(v_{0}\right)\right)=0$. Since $\omega\left(v_{0}, v_{0}\right)=0$, and $T\left(v_{0}\right)$ is closed, it follows from Lemma 1.5 that $v_{0} \in T\left(v_{0}\right)$.

We also have the following interesting result by replacing the hypothesis (iii) of Theorem 2.1 with another natural condition.

Theorem 2.2. Suppose that all the hypotheses of Theorem 2.1 except (iii) hold. Assume that

$$
\inf \{\omega(x, v)+\omega(x, T(x)): x \in X\}>0
$$

for every $v \in X$ with $v \notin T(v)$. Then $\operatorname{Fix}(T) \neq \emptyset$.

Proof. Following the proof of Theorem 2.1, there exists a Cauchy sequence $\left\{x_{n}\right\}$ with $x_{n} \in$ $T\left(x_{n-1}\right)$. Due to the completeness of $X$, there exists $v_{0} \in X$ such that $\lim _{n \rightarrow \infty} x_{n}=v_{0}$. Since $\omega(x, \cdot)$ is lower semicontinuous and $x_{m} \rightarrow v_{0} \in X$, it follows for all $n \geq n_{0}$

$$
\begin{gathered}
\omega\left(x_{n}, v_{0}\right) \leq \lim _{m \rightarrow \infty} \inf \omega\left(x_{n}, x_{m}\right) \leq\left(\frac{2 q^{n-n_{0}}}{1-q}\right) \omega\left(x_{n_{0}}, T\left(x_{n_{0}}\right)\right), \\
\omega\left(x_{n}, T\left(x_{n}\right)\right) \leq \omega\left(x_{n}, x_{n+1}\right) \leq 2 q^{n-n_{0}} \omega\left(x_{n_{0}}, T\left(x_{n_{0}}\right)\right) .
\end{gathered}
$$

Assume that $v_{0} \notin T\left(v_{0}\right)$. Then, we have

$$
\begin{aligned}
0 & <\inf \left\{\omega\left(x, v_{0}\right)+\omega(x, T(x)): x \in X\right\} \\
& \leq \inf \left\{\omega\left(x_{n}, v_{0}\right)+\omega\left(x_{n}, T\left(x_{n}\right)\right): n \geq n_{0}\right\} \\
& \leq \inf \left\{\left(\frac{2 q^{n-n_{0}}}{1-q}\right) \omega\left(x_{n_{0}}, T\left(x_{n_{0}}\right)\right)+2 q^{n-n_{0}} \omega\left(x_{n_{0}}, T\left(x_{n_{0}}\right)\right): n \geq n_{0}\right\} \\
& =\frac{2(2-q)}{(1-q) q^{n_{0}}} \omega\left(x_{n_{0}}, T\left(x_{n_{0}}\right)\right) \inf \left\{q^{n}: n \geq n_{0}\right\}=0,
\end{aligned}
$$

which is impossible and hence $v_{0} \in \operatorname{Fix}(T)$.

Now, we present an improved version of Ciric [4, Theorem 6] and which also generalizes due to Latif and Abdou [10, Theorem 1.6] and due to Klim and Wardowski [3, Theorem 1.3].

Theorem 2.3. Let $(X, d)$ be a complete metric space with a w-distance $\omega$. Let $T: X \rightarrow C l(X)$, be a multivalued map. Assume that the following condition hold:

(i) there exist functions $\varphi:[0, \infty) \rightarrow(0,1)$ and $\mu:[0, \infty) \rightarrow[b, 1)$, with $b>0, \mu$ nondecreasing such that

$$
\varphi(t)<\mu(t), \quad \limsup _{r \rightarrow t^{+}} \varphi(r)<\limsup _{r \rightarrow t^{+}} \mu(r),
$$


(ii) for any $x \in X$, there exists $y \in T(x)$ satisfying the following conditions:

$$
\begin{aligned}
& \mu(\omega(x, y)) \omega(x, y) \leq \omega(x, T(x)), \\
& \omega(y, T(y)) \leq \varphi(\omega(x, y)) \omega(x, y)
\end{aligned}
$$

(iii) the map $f: X \rightarrow \mathbb{R}$, defined by $f(x)=\omega(x, T(x))$ is lower semicontinuous.

Then there exists $v_{0} \in X$ such that $f\left(v_{0}\right)=0$. Further if $\omega\left(v_{0}, v_{0}\right)=0$, then $v_{0} \in T\left(v_{0}\right)$.

Proof. Let $x_{0}$ be an arbitrary, then there exists $x_{1} \in T\left(x_{0}\right)$ such that

$$
\begin{aligned}
& \mu\left(\omega\left(x_{0}, x_{1}\right)\right) \omega\left(x_{0}, x_{1}\right) \leq \omega\left(x_{0}, T\left(x_{0}\right)\right), \\
& \omega\left(x_{1}, T\left(x_{1}\right)\right) \leq \varphi\left(\omega\left(x_{0}, x_{1}\right)\right) \omega\left(x_{0}, x_{1}\right) .
\end{aligned}
$$

From (2.48) we have

$$
\omega\left(x_{1}, T\left(x_{1}\right)\right) \leq \frac{\varphi\left(\omega\left(x_{0}, x_{1}\right)\right)}{\mu\left(\omega\left(x_{0}, x_{1}\right)\right)} \omega\left(x_{0}, T\left(x_{0}\right)\right) .
$$

Define a function $\psi:[0, \infty) \rightarrow[0, \infty)$ by

$$
\psi(t)=\frac{\varphi(t)}{\mu(t)} \quad \forall t \in[0, \infty)
$$

Since $\varphi(t)<\mu(t)$, we have

$$
\begin{gathered}
\psi(t)<1, \\
\limsup _{r \rightarrow t^{+}} \psi(r)<1 \quad \forall t \in[0, \infty) .
\end{gathered}
$$

Thus from (2.49)

$$
\omega\left(x_{1}, T\left(x_{1}\right)\right) \leq \psi\left(\omega\left(x_{0}, x_{1}\right)\right) \omega\left(x_{0}, T\left(x_{0}\right)\right) .
$$

Similarly, there exists $x_{2} \in T\left(x_{1}\right)$ such that

$$
\begin{aligned}
& \mu\left(\omega\left(x_{1}, x_{2}\right)\right) \omega\left(x_{1}, x_{2}\right) \leq \omega\left(x_{1}, T\left(x_{1}\right)\right), \\
& \omega\left(x_{2}, T\left(x_{2}\right)\right) \leq \varphi\left(\omega\left(x_{1}, x_{2}\right)\right) \omega\left(x_{1}, x_{2}\right) .
\end{aligned}
$$

Then by definition of $\psi$, we get

$$
\omega\left(x_{2}, T\left(x_{2}\right)\right) \leq \psi\left(\omega\left(x_{1}, x_{2}\right)\right) \omega\left(x_{1}, T\left(x_{1}\right)\right) .
$$


Fixed Point Theory and Applications

Continuing this process, we get an orbit $\left\{x_{n}\right\}$ of $T$ at $x_{0}$ such that

$$
\begin{gathered}
\mu\left(\omega\left(x_{n}, x_{n+1}\right)\right) \omega\left(x_{n}, x_{n+1}\right) \leq \omega\left(x_{n}, T\left(x_{n}\right)\right), \\
\omega\left(x_{n+1}, T\left(x_{n+1}\right)\right) \leq \varphi\left(\omega\left(x_{n}, x_{n+1}\right)\right) \omega\left(x_{n}, x_{n+1}\right) .
\end{gathered}
$$

Thus

$$
\omega\left(x_{n+1}, T\left(x_{n+1}\right)\right) \leq \psi\left(\omega\left(x_{n}, x_{n+1}\right)\right) \omega\left(x_{n}, T\left(x_{n}\right)\right) .
$$

Since $\psi(t)<1$ for all $t \in[0, \infty)$, we get

$$
\omega\left(x_{n+1}, T\left(x_{n+1}\right)\right)<\omega\left(x_{n}, T\left(x_{n}\right)\right) .
$$

Thus the sequence of nonnegative real numbers $\left\{\omega\left(x_{n}, T\left(x_{n}\right)\right)\right\}$ is decreasing and bounded below, thus convergent. Now, we want to show that the sequence $\left\{\omega\left(x_{n}, x_{n+1}\right)\right\}$ is also decreasing. Suppose to the contrary, that $\omega\left(x_{n}, x_{n+1}\right) \leq \omega\left(x_{n+1}, x_{n+2}\right)$, then as $\mu(t)$ is nondecreasing, we have

$$
\mu\left(\omega\left(x_{n}, x_{n+1}\right)\right) \leq \mu\left(\omega\left(x_{n+1}, x_{n+2}\right)\right),
$$

Now using (2.56), (2.57) and (2.60) with $n=n+1$, we get

$$
\begin{aligned}
\omega\left(x_{n+1}, x_{n+2}\right) & \leq \frac{\varphi\left(\omega\left(x_{n}, x_{n+1}\right)\right)}{\mu\left(\omega\left(x_{n+1}, x_{n+2}\right)\right)} \omega\left(x_{n}, x_{n+1}\right) \\
& \leq \frac{\varphi\left(\omega\left(x_{n}, x_{n+1}\right)\right)}{\mu\left(\omega\left(x_{n}, x_{n+1}\right)\right)} \omega\left(x_{n}, x_{n+1}\right) \\
& <\psi\left(\omega\left(x_{n}, x_{n+1}\right)\right) \omega\left(x_{n}, x_{n+1}\right) \\
& <\omega\left(x_{n}, x_{n+1}\right),
\end{aligned}
$$

a contradiction. Thus the sequences $\left\{\omega\left(x_{n}, x_{n+1}\right)\right\}$ is decreasing. Now let

$$
\limsup _{n \rightarrow \infty} \psi\left(\omega\left(x_{n}, x_{n+1}\right)\right)=\alpha
$$

Thus by (2.52), $\alpha<1$. Then for any $q \in(\alpha, 1)$, there exists $n_{0} \in \mathbb{N}$ such that

$$
\psi\left(\omega\left(x_{n}, x_{n+1}\right)\right)<q \quad \forall n \geq n_{0} .
$$

So, from (2.58), for all $n \geq n_{0}$, we get

$$
\omega\left(x_{n+1}, T\left(x_{n+1}\right)\right)<q \omega\left(x_{n}, T\left(x_{n}\right)\right) .
$$


Thus by induction we get for all $n \geq n_{0}$

$$
\omega\left(x_{n+1}, T\left(x_{n+1}\right)\right) \leq q^{n+1-n_{0}} \omega\left(x_{n_{0}}, T\left(x_{n_{0}}\right)\right) .
$$

Since $\mu(t) \geq b$, from (2.56) and (2.65), we have

$$
\omega\left(x_{n}, x_{n+1}\right) \leq \frac{1}{b} \omega\left(x_{n}, T\left(x_{n}\right)\right) \leq \frac{1}{b} q^{n-n_{0}} \omega\left(x_{n_{0}}, T\left(x_{n_{0}}\right)\right),
$$

for all $n \geq n_{0}$. Note that $\omega\left(x_{n}, T\left(x_{n}\right)\right) \rightarrow 0$. Now, we show that $\left\{x_{n}\right\}$ is a Cauchy sequence. For all $m>n \geq n_{0}$, we have

$$
\begin{aligned}
\omega\left(x_{n}, x_{m}\right) & \leq \sum_{k=n}^{m-1} \omega\left(x_{k}, x_{k+1}\right) \\
& \leq \frac{1}{b} \sum_{k=n}^{m-1} q^{k-n_{0}} \omega\left(x_{n_{0}}, T\left(x_{n_{0}}\right)\right) \\
& \leq \frac{1}{b}\left(\frac{q^{n-n_{0}}}{1-q}\right) \omega\left(x_{n_{0}}, T\left(x_{n_{0}}\right)\right) .
\end{aligned}
$$

Thus we conclude that $\left\{x_{n}\right\}$ is a Cauchy sequence. Now, proceeding the proof of Theorem 2.1, we get some $v_{0} \in X$ such that $f\left(v_{0}\right)=\omega\left(v_{0}, T\left(v_{0}\right)\right)=0$ and $v_{0} \in T\left(v_{0}\right)$.

Following the proof of Theorem 2.2, we can obtain the following result.

Theorem 2.4. Suppose that all the hypotheses of Theorem 2.3 except (iii) hold. Assume that

$$
\inf \{\omega(x, v)+\omega(x, T(x)): x \in X\}>0
$$

for every $v \in X$ with $v \notin T(v)$. Then $\operatorname{Fix}(T) \neq \emptyset$.

Now, we present a result which is a generalization of Theorem 1.4 due to Klim and Wardowski [3] and Ciric [4, Theorem 7].

Theorem 2.5. Let $(X, d)$ be a complete metric space with a w-distance $\omega$. Let $T: X \rightarrow C l(X)$ be a multivalued map. Assume that the following conditions hold:

(i) there exists a function $\varphi:[0, \infty) \rightarrow[0,1)$ such that for each $t \in[0, \infty)$

$$
\limsup _{r \rightarrow t^{+}} \varphi(r)<1,
$$


Fixed Point Theory and Applications

(ii) for any $x \in X$, there exists $y \in T(x)$ satisfying

$$
\begin{gathered}
\omega(x, y)=\omega(x, T(x)), \\
\omega(y, T(y)) \leq \varphi(\omega(x, y)) \omega(x, y),
\end{gathered}
$$

(iii) the map $f: X \rightarrow \mathbb{R}$ defined by $f(x)=\omega(x, T(x))$ is lower semicontinuous.

Then there exists $v_{0} \in X$ such that $f\left(v_{0}\right)=0$. Further if $\omega\left(v_{0}, v_{0}\right)=0$, then $v_{0} \in T\left(v_{0}\right)$.

Proof. Let $x_{0} \in X$, be any initial point. Then from (ii) we can choose $x_{1} \in T\left(x_{0}\right)$ such that

$$
\begin{gathered}
\omega\left(x_{0}, x_{1}\right)=\omega\left(x_{0}, T\left(x_{0}\right)\right) \\
\omega\left(x_{1}, T\left(x_{1}\right)\right) \leq \varphi\left(\omega\left(x_{0}, x_{1}\right)\right) \omega\left(x_{0}, x_{1}\right) \quad \varphi\left(\omega\left(x_{0}, x_{1}\right)\right)<1 .
\end{gathered}
$$

Using the analogous method like in the proof of Lemma 2.1 [10], we obtain the existence of Cauchy sequence $\left\{x_{n}\right\}$ such that $x_{n} \in T\left(x_{n-1}\right)$ and satisfying

$$
\begin{gathered}
\omega\left(x_{n}, x_{n+1}\right)=\omega\left(x_{n}, T\left(x_{n}\right)\right), \\
\omega\left(x_{n+1}, T\left(x_{n+1}\right)\right) \leq \varphi\left(\omega\left(x_{n}, x_{n+1}\right)\right) \omega\left(x_{n}, x_{n+1}\right), \quad \varphi\left(\omega\left(x_{n}, x_{n+1}\right)\right)<1 .
\end{gathered}
$$

Consequently, there exists $v_{0} \in X$ such that $\lim _{n \rightarrow \infty} x_{n}=v_{0}$. Since $f$ is lower semicontinuous, we have

$$
0 \leq f\left(v_{0}\right) \leq \liminf _{n \rightarrow \infty} f\left(x_{n}\right)=0,
$$

thus, $f\left(v_{0}\right)=\omega\left(v_{0}, T\left(v_{0}\right)\right)=0$. Further by closedness of $T\left(v_{0}\right)$ and since $\omega\left(v_{0}, v_{0}\right)=0$, it follows from Lemma 1.5 that $v_{0} \in T\left(v_{0}\right)$.

\section{Examples}

The following example shows that Theorem 2.1 is a genuine generalization of Ciric [4, Theorem 5].

Example 3.1. Let $X=[0,1]$ with the usual metric $d$. Define a function $\omega: X \times X \rightarrow[0, \infty)$, by

$$
\omega(x, y)=y \quad \forall x, y \in X
$$

Clearly, $\omega$ is a $w$-distance on $X$ and $\omega \neq d$. Let $T: X \rightarrow C l(X)$ be such that

$$
T(x)= \begin{cases}\left\{\frac{1}{2} x^{2}\right\}, & \text { for } x \in\left[0, \frac{15}{32}\right) \cup\left(\frac{15}{32}, 1\right], \\ \left\{\frac{17}{96}, \frac{1}{4}\right\}, & \text { for } x=\frac{15}{32} .\end{cases}
$$


Define now $\varphi:[0, \infty) \rightarrow[0,1)$ as follows

$$
\varphi(t)= \begin{cases}\frac{8}{5} t, & \text { for } t \in\left[0, \frac{1}{2}\right) \\ \frac{4}{5}, & \text { for } t \in\left[\frac{1}{2}, \infty\right)\end{cases}
$$

Note that

$$
f(x)=\omega(x, T(x))= \begin{cases}\frac{1}{2} x^{2}, & \text { for } x \in\left[0, \frac{15}{32}\right) \cup\left(\frac{15}{32}, 1\right] \\ \frac{17}{96}, & \text { for } x=\frac{15}{32}\end{cases}
$$

and $f$ is lower semicontinuous. Moreover for each $x \in[0,15 / 32) \cup(15 / 32,1]$ we have $T(x)=$ $\left\{(1 / 2) x^{2}\right\}$. Take $y=(1 / 2) x^{2}$, then we have

$$
\omega(x, y)=\omega(x, T(x))=\omega\left(x, \frac{1}{2} x^{2}\right)=\frac{1}{2} x^{2}
$$

Further, note that

$$
\begin{gathered}
\omega(x, y)=\frac{1}{2} x^{2} \leq[2-\varphi(\omega(x, y))] \frac{1}{2} x^{2}=[2-\varphi(\omega(x, y))] \omega(x, T(x)) \\
\omega(y, T(y))=\omega\left(\frac{1}{2} x^{2}, \frac{1}{8} x^{4}\right)=\left(\frac{1}{4} x^{2}\right) \omega(x, y)<\frac{8}{5}\left(\frac{1}{2} x^{2}\right) \omega(x, y)=\varphi(\omega(x, y)) \omega(x, y) .
\end{gathered}
$$

Hence, for all $x \in[0,1], x \neq 15 / 32, T$ satisfies all the conditions of Theorem 2.1. Now, if $x=$ $15 / 32$, then we have $T(x)=\{17 / 96,1 / 4\}$, and

$$
\omega(x, T(x))=\omega\left(\frac{15}{32},\left\{\frac{17}{96}, \frac{1}{4}\right\}\right)=\inf \left\{\frac{17}{96}, \frac{1}{4}\right\}=\frac{17}{96}
$$

Note that for $x=15 / 32$ there is $y=17 / 96 \in T(x)$ such that

$$
\begin{aligned}
\omega(x, y) & =\frac{17}{96}<\left[2-\frac{8}{5}\left(\frac{17}{96}\right)\right] \frac{17}{96}=[2-\varphi(\omega(x, y))] \omega(x, y), \\
\omega(y, T(y)) & =\omega\left(\frac{17}{96}, \frac{1}{2}\left(\frac{17}{96}\right)^{2}\right)=\frac{1}{2}\left(\frac{17}{96}\right)^{2} \\
& <\frac{8}{5}\left(\frac{17}{96}\right)\left(\frac{17}{96}\right)=\varphi(\omega(x, y)) \omega(x, y) .
\end{aligned}
$$


Thus, $T$ also satisfies all the conditions of Theorem 2.1 for $x=15 / 32$. Hence it follows from Theorem 2.1 that $\operatorname{Fix}(T) \neq \emptyset$. Note that $\operatorname{Fix}(T)=\{0\}$. Clearly, $T$ does not satisfy the hypotheses of Ciric [4, Theorem 5] because $\omega$ is not the metric $d$.

Finally, we present an example which shows that Theorem 2.5 is a genuine generalization of Theorem 1.4 due to Klim-Wardowski [3].

Example 3.2. Let $X=[0, \infty)$ with the usual metric $d$. Define a function $\omega: X \times X \rightarrow[0, \infty)$, by

$$
\omega(x, y)=x+y \quad \forall x, y \in X
$$

Then $\omega$ is a $w$-distance on $X$. Note that $\omega \neq d$. Now, for any real number $a>1$, define $T: X \rightarrow$ $\mathrm{Cl}(\mathrm{X})$ by

$$
T(x)=\left\{\frac{x}{a}\right\} \cup[(1+2 x), \infty), \quad \forall x \in[0, \infty),
$$

and define a constant function $\varphi:[0, \infty) \rightarrow[0,1)$ by

$$
\varphi(t)=\frac{1}{a}, \quad \forall t \in[0, \infty)
$$

Note that $\varphi(t)<1$ for all $t \in[0, \infty)$. And for each $x \in X$ we have

$$
f(x)=\omega(x, T(x))=x+\frac{x}{a}=\left(\frac{a+1}{a}\right) x
$$

Thus, $f$ is continuous. Now for each $x \in[0, \infty)$ there exists $y=(x / a) \in T(x)$ satisfying

$$
\begin{gathered}
\omega(x, y)=\omega\left(x, \frac{x}{a}\right)=\omega(x, T(x)) \\
\omega(y, T(y))=\frac{x}{a}+\frac{x}{a^{2}}=\frac{1}{a}\left(\frac{a+1}{a}\right) x=\varphi(\omega(x, y)) \omega(x, y) .
\end{gathered}
$$

Therefore, all assumptions of Theorem 2.5 are satisfied and $\operatorname{Fix}(T)=\{0\}$. Note that $T(x)$ is not compact for all $x \in X$ and the $w$-distance $\omega$ is not a metric $d$, so $T$ do not satisfy the hypotheses of Theorem 1.4.

\section{Acknowledgment}

The authors thank the referees for their valuable comments. 


\section{References}

[1] S. B. Nadler Jr., "Multi-valued contraction mappings," Pacific Journal of Mathematics, vol. 30, pp. 475$488,1969$.

[2] Y. Feng and S. Liu, "Fixed point theorems for multi-valued contractive mappings and multi-valued Caristi type mappings," Journal of Mathematical Analysis and Applications, vol. 317, no. 1, pp. 103-112, 2006.

[3] D. Klim and D. Wardowski, "Fixed point theorems for set-valued contractions in complete metric spaces," Journal of Mathematical Analysis and Applications, vol. 334, no. 1, pp. 132-139, 2007.

[4] L. Ciric, "Fixed point theorems for multi-valued contractions in complete metric spaces," Journal of Mathematical Analysis and Applications, vol. 348, no. 1, pp. 499-507, 2008.

[5] N. Mizoguchi and W. Takahashi, "Fixed point theorems for multivalued mappings on complete metric spaces," Journal of Mathematical Analysis and Applications, vol. 141, no. 1, pp. 177-188, 1989.

[6] O. Kada, T. Suzuki, and W. Takahashi, "Nonconvex minimization theorems and fixed point theorems in complete metric spaces," Mathematica Japonica, vol. 44, no. 2, pp. 381-391, 1996.

[7] W. Takahashi, Nonlinear Functional Analysis: Fixed Point Theory and Its Application, Yokohama Publishers, Yokohama, Japan, 2000.

[8] L.-J. Lin and W.-S. Du, "Some equivalent formulations of the generalized Ekeland's variational principle and their applications," Nonlinear Analysis: Theory, Methods E Applications, vol. 67, no. 1, pp. 187-199, 2007.

[9] A. Latif and W. A. Albar, "Fixed point results in complete metric spaces," Demonstratio Mathematica, vol. 41, no. 1, pp. 145-150, 2008.

[10] A. Latif and A. A. N. Abdou, "Fixed points of generalized contractive maps," Fixed Point Theory and Applications, vol. 2009, Article ID 487161, 9 pages, 2009. 